\title{
SOBRE LOS ALGORITMOS DE PLANIFICACIÓN DEL MOVIMIENTO DE ROBOTS
}

\author{
José María Bañón* \\ Olmedo Arcila** \\ Jaime Arango ${ }^{\star \star \star}$
}

* Doctor de Estado. Profesor Titular de la Universidad del Valle.

** Profesor Auxiliar de la Universidad del Valle.

*** Ph.D. Profesor del Departamento de Matemáticas de la Universidad del Valle.

\section{RESUMEN}

El objeto de este artículo es doble. Por una parte se describen los algoritmos utilizados en planificación de movimiento de robots, y por otra, se presentan los primeros resultados del desarrollo de un algoritmo completo de movimiento de dos robots circulares e independientes. El trabajo es el resultado de un proyecto financiado por COLCIENCIAS y realizado conjuntamente por el Departamento de Ciencias de la Computación y el Departamento de Matemáticas. 


\section{ABSTRACT}

This article has a two-fold purpose. By one respect, it is devoted to the review of the algorithms employed in robot motion planning. By another respect, it presents the first outcomes from developing a complete algorithm for the motion of two independent circular robots. This work is the result of a project supported by COLCIENCIAS and carried out together by the Computing Science and the Mathematics Department.

\section{QUÉ ES LA PLANIFICACIÓN DEL MOVIMIENTO?}

La investigación en robótica en los últimos 20 años [1][2][3] ha estado fuertemente dirigida hacia el desarrollo de robots autónomos y móviles, es decir de robots capaces de decidir su propio movimiento con el propósito de ejecutar una determinada tarea con objetos en un espacio de trabajo. Una tarea típica consiste por ejemplo en transportar ciertos objetos desde un lugar inicial hasta su destino final. Para la realización de la tarea el robot debe ser capaz de encontrar un camino que evite todo tipo de colisiones con los objetos del espacio de trabajo. Evidentemente, el robot debe razonar acerca de la geometría del espacio de trabajo y de los objetos del mismo. La planificación del movimiento de robots trata del desarrollo de algoritmos geométricos eficientes que doten al robot con la capacidad de generar caminos libres de colisión.

En este artículo se introduce el problema básico de la planificación del movimiento, se presenta una taxonomía de los diferentes algoritmos de planificación y se describen conceptos generales sobre los mismos. Seguidamente se resumen los principales métodos de planificación con especial énfasis en los métodos completos. Finalmente se presentan resultados experimentales del desarrollo de un planificador completo.

\section{EL PROBLEMA BÁSICO DE LA PLANIFICACIÓN DEL MOVIMIENTO}

El problema básico de la planificación del movimiento de robots consiste en, dadas dos configuraciones de un robot, inicial y final, encontrar un camino libre de colisiones con los objetos del espacio de trabajo que enlace la configuración inicial con la final [2]. En principio, el problema básico asume un perfecto conocimiento de la geometría del espacio de trabajo y supone que los objetos son estacionarios.

El problema básico es el problema más general de planificación del movimiento. Existen muchas extensiones del problema básico. Por ejemplo, los objetos del espacio de trabajo pueden no ser estacionarios y poseer un movimiento propio. El robot puede no tener un conocimiento del espacio de trabajo y necesitar la ayuda de sensores.

Reif [4] [5] mostró que la complejidad computacional del problema básico de la planificación del movimiento de un robot, con objetos en un espacio de configuración de dimensión arbitraria es PSPACE-hard, es decir es un problema intratable. Los problemas de planificación que son extensión del problema básico son aún más complejos. La solución a los diversos problemas de planificación del movimiento está condicionada a un buen conocimiento de las soluciones prácticas del problema básico. La comprensión profunda de los algoritmos que resuelven el problema básico, es indispensable para el desarrollo de algoritmos prácticos para las diversas extensiones del problema básico de planificación.

\section{TAXONOMÍA DE LOS ALGORITMOS DE PLANIFICACIÓN}

La mayor parte de los algoritmos de planificación del movimiento se pueden caracterizar según los siguientes esquemas [2]. 
Algoritmos Completos e Incompletos: Teniendo en cuenta la completitud del algoritmo se clasifican en completos e incompletos. Los algoritmos completos son aquellos que garantizan la búsqueda de un camino al problema de la planificación del movimiento si tal camino existe. De lo contrario el algoritmo retorna que no existe solución. Un algoritmo que no garantice la búsqueda de un camino se denomina incompleto.

Algoritmos Resolución-Completos: Existen algoritmos que trabajan con una resolución arbitrariamente pequeña. Tal es el caso de los algoritmos que utilizan una discretización del espacio de configuración. Si para cada resolución el algoritmo garantiza la búsqueda del camino entonces el algoritmo se clasifica como resolución-completo.

Algoritmos Probabilísticamente Completos: Los algoritmos probabilísticamente completos son aquellos que garantizan la búsqueda de un camino, si tal camino existe, con probabilidad tendiendo hacia uno [9].

Algoritmos Heurísticos: Debido a que los algoritmos completos son en general computacionalmente prohibitivos por su enorme complejidad, han sido desarrollados muchos algoritmos que incluyen heurísticas con el objeto de acelerar la búsqueda del camino [17].

Algoritmos Globales y Locales: Dependiendo de la cantidad de información que se utilice para la planificación del movimiento, el algoritmo puede clasificarse en global y local. Si se utiliza todo el espacio de trabajo del robot, el algoritmo es global. Si únicamente se considera parte del espacio de trabajo del robot el algoritmo es local. Es evidente que un algoritmo completo es siempre global, puesto que para garantizar la búsqueda de un camino es necesario conocer la totalidad de la geometría del espacio de trabajo del robot. Así mismo, un algoritmo local será siempre
Incompleto.

\section{DEFINICIONES Y GENERALIDADES}

Un concepto importante en robótica es el espacio de configuración Cspace del robot. Una configuración q de un robot consiste en el conjunto del mínimo número de coordenadas que determinan completamente la posición de un robot; son en general coordenadas cartesianas o articulares, es decir, longitudes o ángulos. El espacio de configuración del sistema Cspace es un espacio de dimensión $n$; consiste en el conjunto de todas las configuraciones permitidas del sistema, donde $n$ es el número de grados de libertad del sistema.

Los obstáculos en el espacio de trabajo del robot llevados al Cspace se denominan Cobstáculos. El conjunto unión de todos los C-obstáculos lo notaremos por Cobst y representa todas aquellas configuraciones del robot que significan colisiones entre obstáculos y el robot. El espacio-libre Cfree es el espacio complementario del Cobst. En general el conjunto Cspace de un robot, con $n$ grados de libertad es un espacio n-dimensional. Los subconjuntos Cfree y Cobst son volúmenes $\mathrm{n}$ dimensionales.

El problema de la planificación de un camino para un robot con $n$ grados de libertad puede ser visto como el problema equivalente de planificar un camino en el espacio-libre Cfree que una la configuración inicial con la final.

\section{CLASIFICACIÓN DE LOS MÉTODOS DE PLANIFICACIÓN}

Los diferentes métodos de planificación del movimiento de robots pueden clasificarse en: método del campo de potencial, método de la descomposición en celdas y métodos de mapas de caminos. 
Métodos del Campo de Potencial

Uno de los métodos más exitosos de planificación del movimiento es el método del campo de potencial. Está inspirado en la mecánica. La idea central desarrollada por Khatib [1], consiste en definir un potencial repulsivo en cada obstáculo y un potencial atractivo en la destinación del robot. Se construye el potencial total resultante como la combinación lineal de todos los potenciales atractivos y repulsivos definidos. El gradiente del potencial resultante representa una fuerza que conduce al robot a su destino. El gran inconveniente del método es que la mayoría de los potenciales utilizados introducen mínimos locales que conducen a posiciones en las cuales el robot queda bloqueado. Sacar al robot de esos mínimos locales requiere tomar decisiones que dependen de la posición particular y esto limita la aplicabilidad del método del campo de potencial. Muchos trabajos han estado dirigidos a la obtención de potenciales sin mínimos locales [6] [7] [8].

\section{Métodos de Descomposición en Celdas}

La descomposición en celdas representa una gran variedad de métodos cuyo fundamento matemático consiste en la descomposición del conjunto de configuraciones libres en un conjunto de regiones disjuntas, denominadas celdas [3]. La descomposición puede ser exacta $\mathrm{O}$ aproximada. Las relaciones de adyacencia entre las celdas se representan en el grafo de conectividad, el cual captura la conectividad del espacio libre. El problema de la búsqueda de un camino contínuo y libre de colisiones se reduce al problema discreto de encontrar un camino en el grafo de conectividad del sistema. En general, estos métodos conducen a un preprocesamiento largo y rápidamente llega a ser intratable para números de grados de libertad elevados.

Una variante muy utilizada de este método es la descomposición jerárquica en celdas [10] [1 1]. Consiste en descomponer el espacio de configuraciones del robot en celdas rectangulares en una jerarquía de niveles de aproximación, de manera incremental, y con la resolución que interesa.

\section{Métodos de Mapas de Caminos}

También denominados métodos de retracción, estos métodos capturan la conectividad del espacio libre mediante una red de curvas o mapa de caminos. Entonces, la búsqueda de un camino que una la configuración inicial y final se reduce a la búsqueda en el mapa de un camino. La dificultad principal de estos métodos consiste en la construcción de la red de curvas. Entre los diferentes métodos de mapas de caminos merecen destacarse: los diagramas de voronoi, la transformada del eje medio, los grafos de visibilidad, las redes de autopistas y el método de la silueta [12] [13].

Uno de los primeros métodos de planificación de caminos en robótica es el método del grafo de visibilidad [12]. En este método se construye un grafo de conectividad en el que las aristas se forman por segmentos rectos que unen vértices de la frontera de la configuración libre.

El método de la silueta [13], resuelve el problema básico de la planificación del movimiento en tiempo simplemente exponencial con respecto a la dimensión del espacio de configuración. Es un algoritmo que se aplica a robots con un número arbitrario de grados de libertad.

\section{ALGORITMOS COMPLETOS DE PLANIFICACIÓN DEL MOVIMIENTO}

Dos tipos de algoritmos completos han sido propuestos en la literatura: algoritmos generales y algoritmos particulares. 
Algoritmos Generales Completos: Resuelven en su generalidad el problema de la planificación del movimiento. Son válidos para cualquier geometría del robot y de su entorno. Schwartz y Sharir [14] desarrollaron un algoritmo general basado en la descomposición exacta del espacio libre en celdas. El algoritmo funciona en tiempo doblemente exponencial con respecto al número de grados de libertad del sistema. En [13] se desarrolla el método de la silueta y funciona en tiempo simplemente exponencial. Debido a su elevada complejidad son muy ineficientes y nunca han sido implementados en la práctica. El interés de los algoritmos generales es que sirven como prueba de la existencia de un método general de planificación del movimiento.

Algoritmos Particulares Completos: LOS algoritmos particulares son desarrollados para geometrías específicas del robot y del entorno. En general funcionan en tiempo polinomial y se aplican a los robots con un número de grados de libertad inferior o igual a 4 . Entre ellos, merecen destacarse los trabajos [15] [16] [17] basados en una descomposición exacta del espacio libre para diversas geometrías particulares de robots. Estos métodos son interesantes para ser aplicados a entornos de trabajo muy reducidos en los cuales se requiere una completa descripción del mismo.

En general han habido muy pocas implementaciones de algoritmos completos. Avnaim et al [18] implementan un algoritmo exacto para planificar el camino de un polígono a través de obstáculos poligonales. En [19] Bañón implementa el algoritmo [18] y demuestra que el algoritmo no era completo como proclamaban sus autores. El algoritmo se extendió para cubrir la totalidad de los casos que podrían presentarse.

\section{El Desarrollo de un Planificador y Resultados}

En esta última sección se exponen los primeros resultados de la implementación de un planificador exacto del movimiento. Presentamos de forma muy resumida los fundamentos del algoritmo.

El Algoritmo: El planificador desarrollado está basado en el algoritmo completo descrito en [16]. Se trata de un algoritmo de planificación para dos robots, $B_{1}$ y $B_{2}$, circulares $e$ independientes en un entorno de trabajo constituido por objetos poligonales y estacionarios en el plano euclídeo. Se asume que los robots conocen perfectamente la geometría del entorno.

El Espacio de Configuración: El espacio de configuración Cspace está formado por las posiciones $\mathrm{P}_{1}$ y $\mathrm{P}_{2}$ de los robots. El sistema tiene cuatro grados de libertad. El espacio-libre Cfree es un hipervolumen de dimensión 4.

Metodología: El algoritmo se basa en la descomposición exacta del espacio libre Cfree en celdas. Para ello se utiliza el método de la proyección que consiste en fijar uno de los robots y estudiar los posibles movimientos del otro robot. Ciertas estructuras de la frontera de Cfree se proyectan bajo la forma de curvas denominadas curvas críticas. Tales curvas dividen al espacio de las posiciones admisibles de uno de los robots en regiones con ciertas propiedades de invarianza lo cual permite la descomposición del espacio-libre en celdas de manera exacta. Cada celda corresponde a una clase determinada de movimiento de los dos robots.

El Grafo de Conectividad: El grafo de conectividad CG captura todos los movimiento posibles del sistema entre dos celdas cualesquiera. Las celdas son los nodos del grafo CG. Dos celdas son adyacentes si existe un camino continuo que las une directamente sin pasar por otra celda intermedia. Las aristas del grafo CG se establecen entre celdas adyacentes.

Búsqueda de Caminos: La planificación del 
movimiento de los cuerpos $B_{1}$ y $B_{2}$ se reduce a encontrar un camino en el grafo CG que una las celdas inicial $\mathrm{Cly}$ final $C F$, es decir en buscar una sucesión de celdas adyacentes $\mathrm{C}_{1}$, $\mathrm{C}_{2}, \ldots, \mathrm{CN}_{\mathrm{N}}$ tales que $\mathrm{Cl}=\mathrm{C}_{1}$ y $\mathrm{CF}=\mathrm{CNN}_{\text {. }} \quad \mathrm{La}$ completitud del algoritmo garantiza que si no se encuentra un camino en el grafo entonces no hay un movimiento libre de colisiones entre la configuración inicial y final. Finalmente se genera la trayectoria de los robots de manera que esté comprendida en los canales encontrados.

Resultados Experimentales: El algoritmo ha sido implementado en lenguaje $\mathrm{C}$ y en una estación de trabajo Sun. Debido a los numerosos errores que surgen en el procesamiento de objetos geométricos, se ha puesto especial cuidado en los cálculos. Se ha trabajado con factores de tolerancia [24]. como ilustración de la implementación, hemos escogido el caso de dos robots confinados en el interior de un heptágono como se muestra en la figura 1. Este es un caso particularmente interesante debido a que el espacio de trabajo es muy estrecho por lo que la planificación del movimiento resulta difícil. La figura 1 muestra la sucesión coordinada de movimientos necesarios para ir de la configuración inicial a la final. En el interior del heptágono los dos robots circulares alternan sus movimientos para alcanzar el objeto final.

Merece señalarse que son muy pocos los algoritmos completos para dos robots que han sido implementados [20]. El análisis del algoritmo así como los detalles de la implementación serán publicados próximamente.

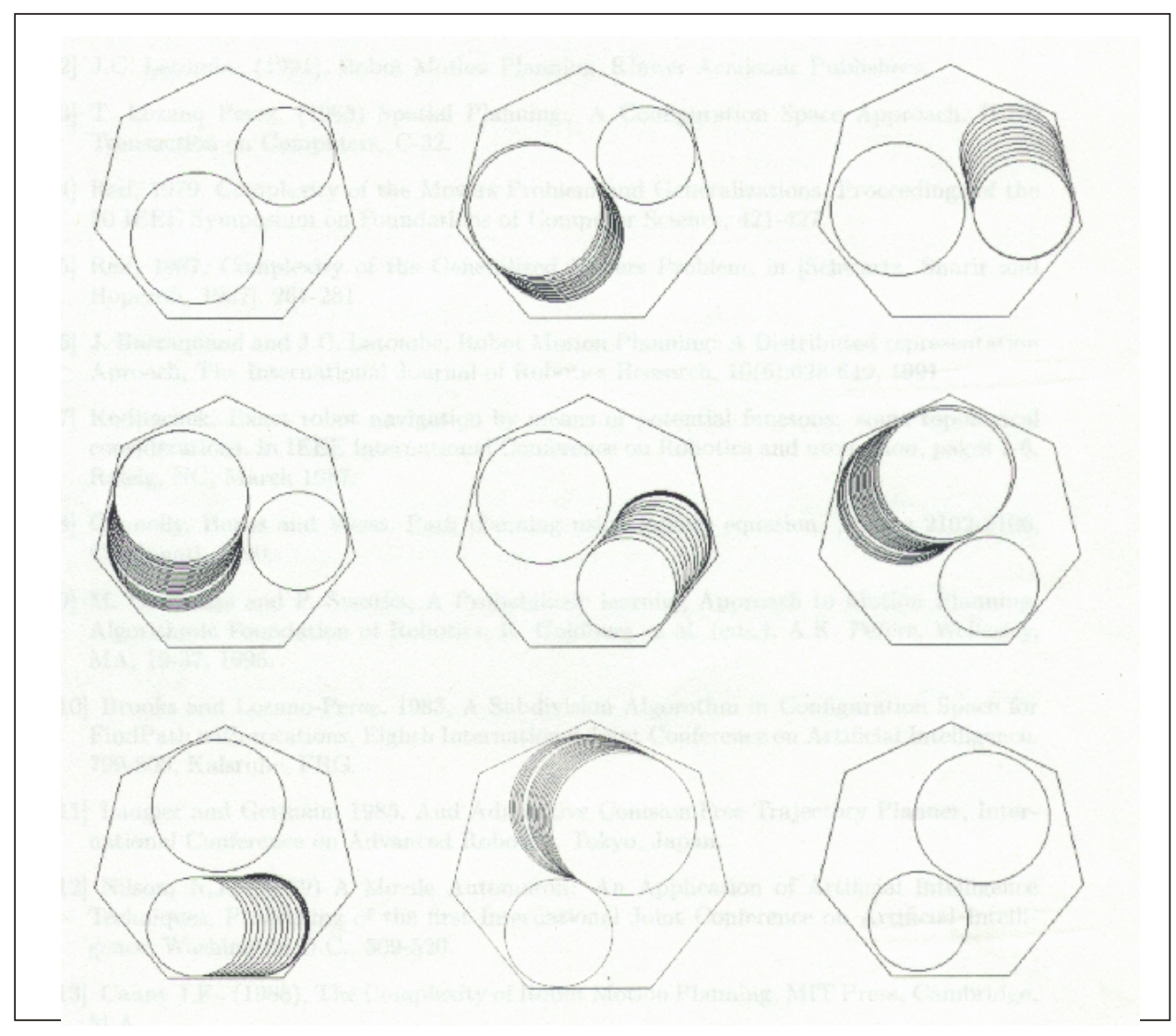

Figura 1. Planificación del Movimiento 


\section{BIBLIOGRAFÍA}

1] O. Khatib (1986), Real-Time Obstacle Avoidance for Manipulators and Mobile Robots. Intern. Journal of Robotics Research 5, 1, MIT Press.

[2] J.C. LARTOMBE, (1991), Robot Motion Planning, Kluwer Academic Publishers

[3] T. LOZANO PÉREZ, (1983) Spatial Planning: A Configuration Space Approach. IEEE Transaction on Computers, C-32.

[4] REIF, 1979. Complexity of the Movers Problem and Generalizations, Proccedings of the 20. IEEE Symposium on Foundations of Computer Science, 421-427.

[5] REIF, 1987. Complexity of the Generalized Movers Problem, in [Schwartz, Sharir and Hopcroft, 1987], 267-281.

[6] J. BARRAQUAND AND J.C. LATOMBE, Robot Motion Planning: A Distributed representation Aproach, The International Journal of Robotics Research, 10(6):628- 649, 1991.

[7] KODITSCHEK, Exact robot navigation by means of potential functions: some topological considerations. In IEEE International Conference on Robotics and Automation, pages 1-6, Raleig, NC, March 1987.

[8] CONNOLLY, BURNS AND WEISS. Path planning using laplace equation, pags. 2102 - 2106, Cincinnati, 1990.

[9] M. OVERMARS AND P. SVESTKA, A Probabilistic learning Approach to Motion Planning, Algorithmic Foundation of Robotics, K. Goldberg ef al. (eds.), A. K. Peters, Wellesley, MA, 19-37, 1995.
[10] BROOKS AND LOZANO-PEREZ, 1983, A Subdivision Algorithm in Configuration Space for FindPath with rotations, Eighth International Joint Conference on Artificial Intelligence. 799-806, Kalsruhe. FRG.

[11] LAUGIER AND GERMAIN, 1985, And Adaptative Collision. Free Trajectory Planner, International Conference on Advanced Robotics, Tokyo, Japan.

[12] NILSON, N,J, (1969) A Movile Automaton: An Application of Artificial Intelligence Techniques, Procceding of the first International Joint Conference on Artificial Intelligence, Washington D.C., 509-520.

[13] CANNY J.F., (1988), The Complexity of Robot Motion Planning, MIT Press, Cambridge, M.A.

[14] J.T. SCHWARTZ, M. SHARIR, (1983), On the Piano Movers problem. II. General Techniques for Computing Topological Properties of Real Algebraic Manifolds. Advances in Applied Mathematics, 4, 298-351.

[15] J.T. SCHWARTZ AND M. SHARIR, (1983), On the Piano Movers Problem. I, The case of a Two-Dimensional Rigid Polygonal Body Moving Amidst Polygonal Barriers. Communication on Pure and Applied Mathemathics, 36, 345-398.

[16] J.T. SCHWARTZ AND M. SHARIR, (1983), on the Piano Movers Problem. III, Coordinating the motion of several independent bodies: The special case of two circular bodies moving amidst polygonal barriers. International Journal Robotics Research, 2, 4675. 
[17] D. HALPERIN AND M. SHARIR. A nearquadratic algorithm for planning the motion of a polygon in a polygonal environment. Discrete Comput. Geom., 16:121-134, 1996

[18] Avnain F., Boissonnat J.D. And Faverjon B., 1988, A practical exact motion planning algorithm for polygonal objects amidst polygonal obstacles, Rapports de Recherche 890, INRIA, France.

[19] J. BAÑÓN, (1990), "Implementation and Extension of the Ladder Algorithm", IEEE Proceedings of the International Conference on Robotics and Automation, Ohio, USA.

[20] R. ALAMI, J.P. LAUMOND, and T. SIMEON, Two manipulation planning algorithms, pag. 109-125, Algorithmic Foundation of Robotics, de. A K Peters Ltda., 1995

[21] HART P.E., NILSSON N.J. Y RAPHAEL B., (1968) A formal basis for the heuristic determination of minimun cost paths, IEEE Transactions on Systems, Science and Cybernetics, SSC-4(2), 100107.

[22] J.E. HOPCROFT, J.T. SCHWARTZ, AND M. SHARIR, On the Complexity of Motion Planning for Multiple Independent Objects: PSPACE-Hardness of the Warehouseman Problem, The International Journal of Robotics Research, 3(4):76-88, 1984.

[23] F. LAMIRAUX AND J.P. LAUMOND, On the Expected Complexity of Random Path Planning, rep. No. 95087, LAAS/CNRS, Toulouse, March, 1995.
[[24] S. FANG, B. BRUDERLIN, X ZHU, "Robustness in solid modelling a tolerant based, intuitionistic approach, Computer Aided Design, 25:9, 1993. 